\title{
Synthesis and Characterization of $\operatorname{RuSr}_{2} R_{1.6} \mathrm{Ce}_{0.4} \mathrm{Cu}_{2} \mathrm{O}_{10}(\mathrm{R}=\mathrm{Gd}$, Eu, and Sm) Magnetosuperconductors
}

\author{
Rajneesh Mohan, ${ }^{1}$ Nupinderjeet Kaur, ${ }^{2}$ and N. K. Gaur ${ }^{2}$ \\ ${ }^{1}$ Department of Mechatronics Engineering, Jeju National University, Jeju 690-756, Republic of Korea \\ ${ }^{2}$ Department of Physics, Barkatullah University, Bhopaladhya, Madhya Pradesh 462026, India \\ Correspondence should be addressed to Rajneesh Mohan; rajneesh482@gmail.com
}

Received 4 June 2013; Revised 4 July 2013; Accepted 8 July 2013

Academic Editor: Zigang Deng

Copyright (c) 2013 Rajneesh Mohan et al. This is an open access article distributed under the Creative Commons Attribution License, which permits unrestricted use, distribution, and reproduction in any medium, provided the original work is properly cited.

The discovery of the coexistence of superconductivity and weak ferromagnetism in ruthenocuprates like $\mathrm{RuSr}_{2} \mathrm{R}_{2-x} \mathrm{Ce}_{x} \mathrm{Cu}_{2} \mathrm{O}_{10}\left(\mathrm{Ru}_{\text {, }}\right.$ $\mathrm{R}-1222 ; \mathrm{R}=\mathrm{Eu}, \mathrm{Gd})$ and $\mathrm{RuSr}_{2} \mathrm{GdCu}_{2} \mathrm{O}_{8}(\mathrm{Ru}-1212)$ has created a tremendous interest both experimentally as well as theoretically. Here, we have prepared polycrystalline samples of $\mathrm{RuSr}_{2} \mathrm{R}_{1.4} \mathrm{Ce}_{0.6} \mathrm{Cu}_{2} \mathrm{O}_{10}(\mathrm{R}=\mathrm{Gd}$, Eu, and $\mathrm{Sm})$ by the standard solid state reaction method. These samples were characterized by XRD, SEM, dc resistivity, and squid measurements. All the prepared samples were single phase without any trace of impurity. From the low-temperature resistivity measurement, the samples of $\mathrm{RuSr}_{2} \mathrm{R}_{2-x} \mathrm{Ce}_{x} \mathrm{Cu}_{2} \mathrm{O}_{10}$ $\left(\mathrm{R}=\mathrm{Eu}\right.$ and $\mathrm{Gd}$ ) were found to be superconducting, while the samples of $\mathrm{RuSr}_{2} \mathrm{Sm}_{1.6} \mathrm{Ce}_{0.4} \mathrm{Cu}_{2} \mathrm{O}_{10}$ show semiconducting behavior. The magnetization $(M)$ versus field $(H)$ hysteresis at $5 \mathrm{~K}$ clearly shows the ferromagnetic behavior of the samples. The zero field cooled magnetization $\left(M_{\mathrm{ZFC}}\right)$ and field cooled magnetization $\left(M_{\mathrm{FC}}\right)$ diverge at $100 \mathrm{~K}$.

\section{Introduction}

The discovery of new superconducting and magnetic materials with anomalous properties has revived a significant activity in the field ranging from basic science to developments for technological applications. Usually, it was believed that for conventional superconductors described by the BCS theory [1] superconductivity (SC) and ferromagnetism (FM) are mutually antagonistic phenomena. However, the coexistence of SC and weak FM has been discovered in 1997 in $\mathrm{RuSr}_{2} \mathrm{R}_{2-x} \mathrm{Ce}_{x} \mathrm{Cu}_{2} \mathrm{O}_{10}(\mathrm{R}=\mathrm{Eu}, \mathrm{Gd}, \mathrm{Ru}-1222)$ [2] and subsequently in $\mathrm{RuSr}_{2} \mathrm{GdCu}_{2} \mathrm{O}_{8}(\mathrm{Ru}-1212)[3,4]$. Ru-1222 is called superconducting weak ferromagnet because the SC arises in the FM ordered state [2].

Intensive research has been carried out to understand these magnetosuperconductors, particularly the Ru-1212 [37]. Ru-1212 displays a Curie transition at $T_{M}=132 \mathrm{~K}$ and bulk superconductivity below $0-46 \mathrm{~K}$ depending on the sample preparations $[3,7]$. Superconductivity appears to be associated with the $\mathrm{CuO}_{2}$ planes [6] with some types of long-range magnetic order that involves the $\mathrm{RuO}_{2}$ planes [4] In comparison to $\mathrm{Ru}-1212$, another magnetosuperconductor
Ru-1222 was given less attention [8]. Ru-1222 has a complicated magnetic behavior. It is found to be paramagnetic at room temperature, but as it is cooled down, it undergoes antiferromagnetic transition $[2,8,9]$ followed by spin glass behaviour [10] and ferromagnetic transition $[2,8,9]$. Below the ferromagnetic transition, the superconductivity sets in and coexists with the ferromagnetism. The tetragonal $\mathrm{Ru}-$ 1222 structure evolves from the $\mathrm{RBa}_{2} \mathrm{Cu}_{3} \mathrm{O}_{7}$ (R-123) one by inserting a fluorite-type $\mathrm{R}_{1 . x} \mathrm{Ce}_{x} \mathrm{O}_{2}$ layer instead of single $\mathrm{R}$ layer in $\mathrm{R}-123$. The $\mathrm{Ru}$ ions reside in the $\mathrm{Cu}(1)$ site, and only one distinct $\mathrm{Cu}$ site (corresponding to $\mathrm{Cu}(2)$ in $\mathrm{R}-123$ ) exists. As compared to $\mathrm{Ru}-1212, \mathrm{Ru}-1222$ is very sensitive to oxygen content $[10,11]$. This is the main source responsible for the nonuniform properties. There are great variety of results regarding the influence of the preparation on the $\mathrm{O}$ stoichiometry. The oxygen stoichiometary can be varied with suitable annealing. Some reports suggest that an increase of oxygen content results in the decrease of lattice parameter and increase of $T_{c}[11,12]$. The superconducting transition in this compound is reported in the range $30 \mathrm{~K}-50 \mathrm{~K}$ [13]. The superconducting transition width was found to be very 
broad. The properties (physical, electrical, and magnetic) are strongly "sample dependent."

The study on the ruthenocuprates can throw a light on the nature of superconductivity. Clues to understand the reasons for the contradictory results on superconductivity in this compound can be obtained by having a closer look at the structure and microstructure of the sintered samples.

A variety of experiments can be performed to elucidate some more properties. The effect of annealing in oxidizing/reducing atmosphere and substitution of various cations can provide the clues for understanding the nature of superconductivity in these materials. Still, there is a tremendous scope to improve the materialistic properties of these materials through different types of processing techniques. We have made an attempt to study the effect of annealing on the superconducting properties of $\mathrm{Ru}, \mathrm{R}-1222, \mathrm{R}=\mathrm{Gd}, \mathrm{Eu}$, and Sm.

\section{Experimental}

Polycrystalline samples of $\mathrm{RuSr}_{2} \mathrm{R}_{1.4} \mathrm{Ce}_{0.6} \mathrm{Cu}_{2} \mathrm{O}_{10}$ (where $\mathrm{R}=$ $\mathrm{Gd}, \mathrm{Eu}$, and $\mathrm{Sm}$ ) have been synthesized by solid-state reactions. Starting materials were high purity (99.99\%) $\mathrm{RuO}_{2}$, $\mathrm{SrCO}_{3}, \mathrm{Gd}_{2} \mathrm{O}_{3}$ (or $\mathrm{Eu}_{2} \mathrm{O}_{3}, \mathrm{Sm}_{2} \mathrm{O}_{3}$ ), $\mathrm{CeO}_{2}$, and $\mathrm{CuO}$. These were thoroughly grinded, calcined at $960^{\circ} \mathrm{C}$ in the air, reacted as pellets at $1010^{\circ} \mathrm{C}$ in flowing argon, and then reacted at 1050 and $1060^{\circ} \mathrm{C}$ in flowing oxygen. Each reaction was carried out for $24 \mathrm{~h}$ and, intermediate grinding, and pelletized. Some of the a-sprepared samples were annealed in a quartz tube under a constant flow of high purity oxygen gas at temperature $1060^{\circ} \mathrm{C}$ for $48 \mathrm{~h}$. All the samples in the present investigation were subjected to the structural characterization by X-ray powder diffraction technique (XRD) and low-temperature electrical transport measurement using the standard fourprobe method using a closed-cycle helium cryostat. The grain morphology of the fractured surface of the samples was analyzed by scanning electron microscopy.

\section{Results and Discussion}

The XRD patterns of the $\mathrm{RuSr}_{2} \mathrm{Gd}_{1.4} \mathrm{Ce}_{0.6} \mathrm{Cu}_{2} \mathrm{O}_{10}(\mathrm{Ru}$, Gd-1222), and $\mathrm{RuSr}_{2} \mathrm{Eu}_{1.4} \mathrm{Ce}_{0.6} \mathrm{Cu}_{2} \mathrm{O}_{10}$ (Ru, Eu-1222), $\mathrm{RuSr}_{2} \mathrm{Sm}_{1.4} \mathrm{Ce}_{0.6} \mathrm{Cu}_{2} \mathrm{O}_{10}(\mathrm{Ru}, \mathrm{Sm}-1222)$ are shown in Figure 1. It can be seen from this figure that the samples prepared by us are single phase. The XRD pattern is indexed using CellRef program [14]. The lattice parameters were calculated using the CellRef program in tetragonal space group $I 4 / \mathrm{mmm}$. The calculated lattice parameters of $\mathrm{Ru}, \mathrm{Gd}-1222$ sample are $a=3.832 \AA$ and $c=28.409 \AA$. The obtained value of " $a$ " and " $c$ " coincides with the reported value of Felner et al. $[2,13]$ and Awana et al. [10]. The lattice parameters calculated using CellRef program of $\mathrm{Ru}$, Eu-1222 sample are $a=3.847 \AA$ and $c=28.628 \AA$. The lattice parameters calculated using CellRef program of $\mathrm{Ru}, \mathrm{Sm}-1222$ sample are $a=3.862 \AA$ and $c=28.611 \AA$. The value of lattice parameters " $a$ " for Ru, Gd-1222, Ru, Eu-1222, and Ru, Sm-1222 samples is related to the ionic radii of substituents Gd, Eu and Sm [15]. The bigger substituent ion the larger lattice parameter.

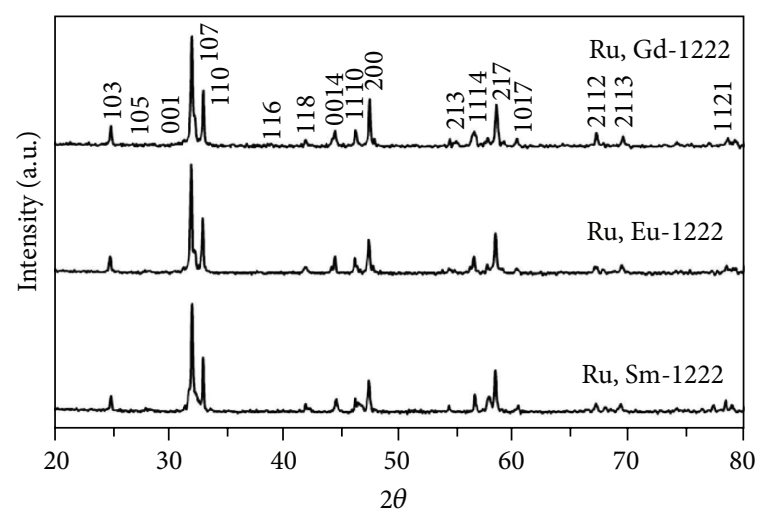

FIGURE 1: XRD pattern of Ru, Gd-1222, Ru, Eu-1222, and Ru, Sm1222.

The SEM images of fractured surface of Ru, Gd-1222, Ru, $\mathrm{Eu}-1222$, and $\mathrm{Ru}, \mathrm{Sm}-1222$ samples are shown in Figure 2. These images clearly show the different grain structure of these compounds. $\mathrm{Ru}, \mathrm{Gd}-1222$ has needles type of grains of different sizes imbedded in each other. $\mathrm{Ru}, \mathrm{Eu}-1222$ has grains with smooth surface. $\mathrm{Ru}, \mathrm{Sm}-1222$ has sponge-type porous grain structures.

The resistivity-temperature behavior $(\rho-T)$ of the asprepared samples of Ru, Gd-1222, Ru, Eu-1222, and Ru, Sm-1222 with corresponding $d \rho / d T$ is shown in Figures 3(a), 3(b), and $3(\mathrm{c})$, respectively.

The asprepared sample of $\mathrm{Ru}, \mathrm{Gd}-1222$ shows metallic normal state resistivity before superconducting transition with onset at $36 \mathrm{~K}$ and $T_{c 0}$ (zero resistivity temperature) at $23 \mathrm{~K}$. The $d \rho / d T$ curve shows the sharper superconducting transition with peak at $25 \mathrm{~K}$ in Figure 3(a). The as-prepared sample of $\mathrm{Ru}, \mathrm{Eu}-1222$ shows semiconducting normal state resistivity before superconducting transition with onset at $28 \mathrm{~K}$ in Figure 3(b). But the zero resistivity could not be observed in this sample down to $13 \mathrm{~K}$. The onset superconducting transition in $\mathrm{Ru}, \mathrm{Eu}-1222$ is also revealed by the $d \rho / d T$ curve. The resistivity behavior of $\mathrm{Ru}, \mathrm{Sm}-1222$ sample is semiconducting type, with no sign of superconductivity observed down to $13 \mathrm{~K}$ as depicted in Figure 3(a). From these results, it may be assumed that superconducting properties of $\mathrm{Ru}-1222$ compound deteriorate with increasing lattice parameters due to the substitution of bigger rare-earth ions (i.e., $\mathrm{Eu}, \mathrm{Sm})$. The bigger ion in $(\mathrm{Eu} / \mathrm{Sm})_{1.6} \mathrm{Ce}_{0.4} \mathrm{O}_{2-\delta}$ plane increases the separation between two $\mathrm{CuO}_{2}$ planes, which are responsible for superconductivity [6]. The $\mathrm{Ru}, \mathrm{Eu}-1222$ sample has low room temperature resistivity than Gd-1222. But the resistivity of Eu-1222 increases progressively with the decreasing temperature in contrast to Gd-1222 sample, in which the resistivity progressively decreases with decreasing temperature.

Since $\mathrm{Ru}, \mathrm{Sm}-1222$ sample does not show any superconducting properties, therefore for magnetic properties study, we concentrate only on Ru, Gd-1222 and Ru, Eu-1222 samples. The static magnetic response of $\mathrm{Ru}, \mathrm{Gd}-1222$ and $\mathrm{Ru}, \mathrm{Eu}-$ 1222 was studied by zero field cooled (ZFC) and field cooled (FC) dc magnetization $\left(M_{\mathrm{ZFC}}\right.$ and $M_{\mathrm{FC}}$, resp.) measured 


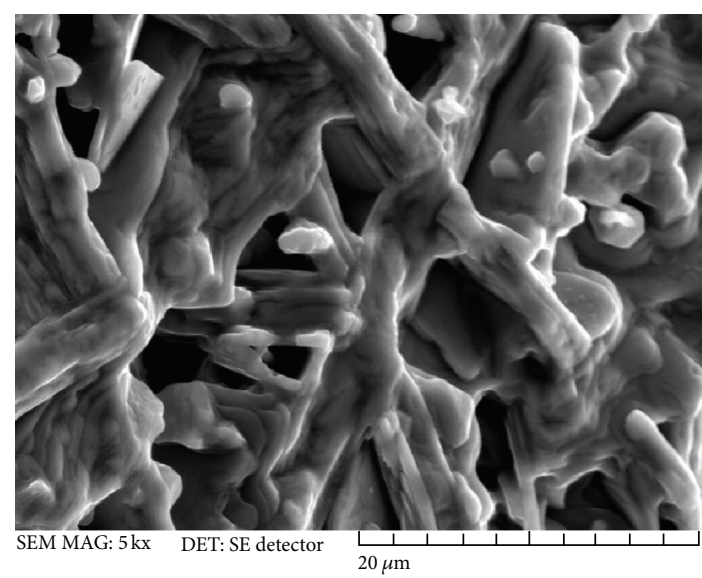

(a)

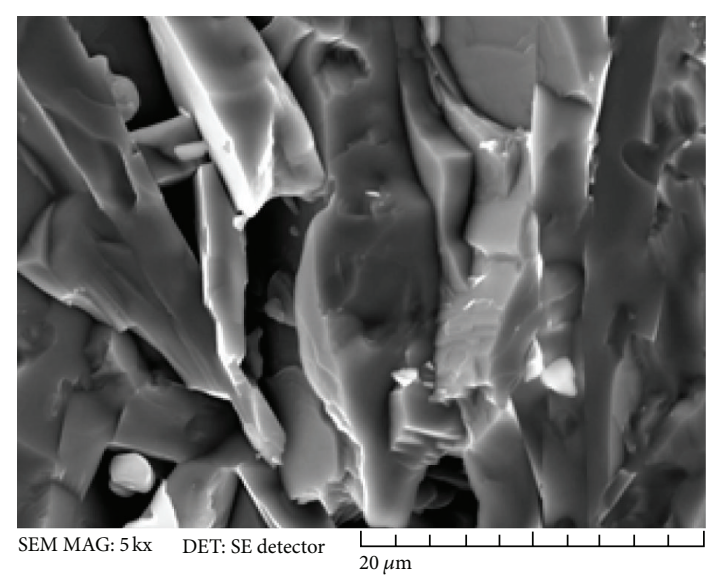

(b)

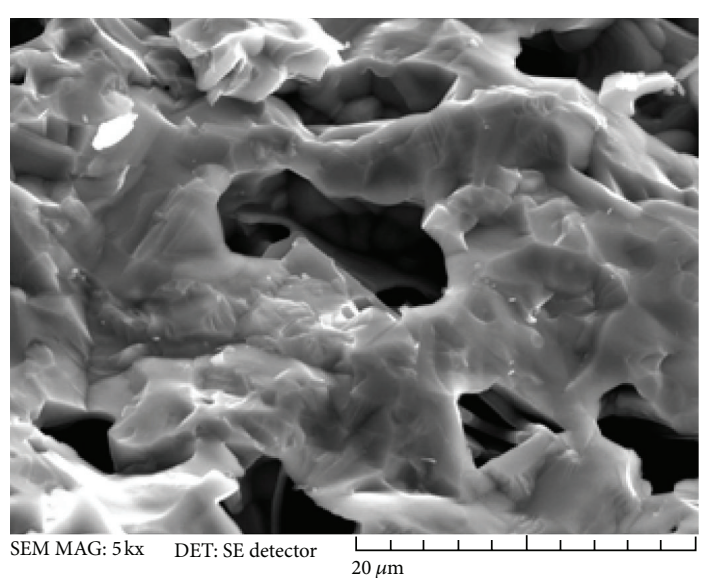

(c)

Figure 2: SEM images of (a) Ru, Gd-1222, (b) Ru, Eu-1222, and (c) Ru, Sm-1222; scale is $20 \mu \mathrm{m}$.

as a function of temperature. Figure 4 shows $M_{\mathrm{ZFC}}$ and $M_{\mathrm{FC}}$ curves as a function of temperature for $100 \mathrm{Oe}$ in the temperature range of 5 to $150 \mathrm{~K}$. The sharp rise of both the $M_{\mathrm{ZFC}}$ and the $M_{\mathrm{FC}}$ curves at $T(\mathrm{~K})=108 \mathrm{~K}$ and $111 \mathrm{~K}$, that is, the Curie temperature $\left(T_{\text {Curie }}\right) \mathrm{Ru}, \mathrm{Gd}-1222$, and $\mathrm{Ru}, \mathrm{Eu}-1222$ respectively, for a PM to FM transition. Here, we have denoted $T_{\text {Curie }}$ as the intercept of the straight line shown in Figure 4 , the $x$-axis. At temperatures close to $T_{\text {Curie }}$, that is, at $T_{\mathrm{irr}}$, the $M_{\mathrm{ZFC}}$, and $M_{\mathrm{FC}}$ curves branch out, and the system enters into a glassy state [16]. The $M_{\mathrm{ZFC}}$ and $M_{\mathrm{FC}}$ versus $T(\mathrm{~K})$ curves exhibit irreversible behavior for temperature below $T_{\text {irr }}$. $T_{\text {irr }}$ for Ru, Gd-1222 and Ru, Eu1222 are $93 \mathrm{~K}$ and $90 \mathrm{~K}$, respectively. The $\mathrm{Ru}$ moments in Ru-1222 order antiferromagnetically at around 125-180 K, which later develops into a canted ferromagnetism due to an antisymmetric exchange coupling of the DzyaloshinskyMoriya type between neighboring $\mathrm{Ru}$ moments, at lower temperatures $80-100 \mathrm{~K}[2]$. Below $T_{\text {irr }}$, the $\mathrm{Ru}-\mathrm{Ru}$ interactions begin to dominate, leading to reorientation of the $\mathrm{Ru}$ moments, which leads to a peak in the magnetization curves [12]. Superconductivity is seen at $5 \mathrm{~K}$ in terms of diamagnetic transition $\left(T_{\text {dia }}\right)$ in applied field of $H=100 \mathrm{Oe}$ (Figure 4$)$. It is known that, due to internal magnetic fields, these compounds are in a spontaneous vortex phase (SVP) even at zero external fields [13]. For $T_{\text {dia }}<T<T_{\mathrm{c}}$, the compound remains in a mixed state. Though $R=0$ is achieved at relatively higher temperatures ( $22 \mathrm{~K}$, see Figure 2), the diamagnetic response is seen at much lower temperatures.

Magnetization $(M)$ versus applied field $(H)$ isotherms at $5 \mathrm{~K}$ for $\mathrm{Ru}, \mathrm{Gd}-1222$ and $\mathrm{Ru}, \mathrm{Eu}-1222$ sample is shown in Figure 5. $M-H$ curves of $\mathrm{Ru}, \mathrm{Gd}-1222$ show FM-like hysteresis with nonsaturating magnetization at high fields (up to $2500 \mathrm{Oe}$ ), suggesting the formation of short-range ordered clusters with FM coupling between the spins of the clusters. In case of $\mathrm{Ru}, \mathrm{Eu}-1222$ sample, the magnetization starts saturating above 1000 Oe field in both directions. In this figure, the coexistence of superconductivity and ferromagnetism is clearly evident. It is clearly visible from the inset given in Figure 5. The hysteresis loop exhibits a Meissner-like linear increase in diamagnetic signal up to 106 and 87 Oe, typical for a superconducting state [16]. The oxygen content in $\mathrm{Ru}-$ 1222-type ruthenocuprate can be varied with annealing. Since the electrical and other properties of cuprates are sensitive to the oxygen content, therefore it becomes pertinent to study the electrical properties of prepared sample subjected to oxygen annealing. 


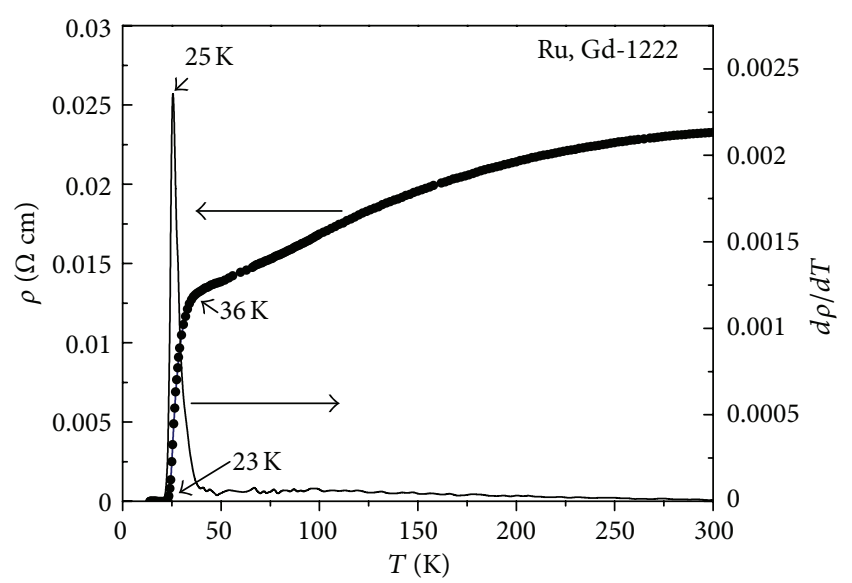

(a)

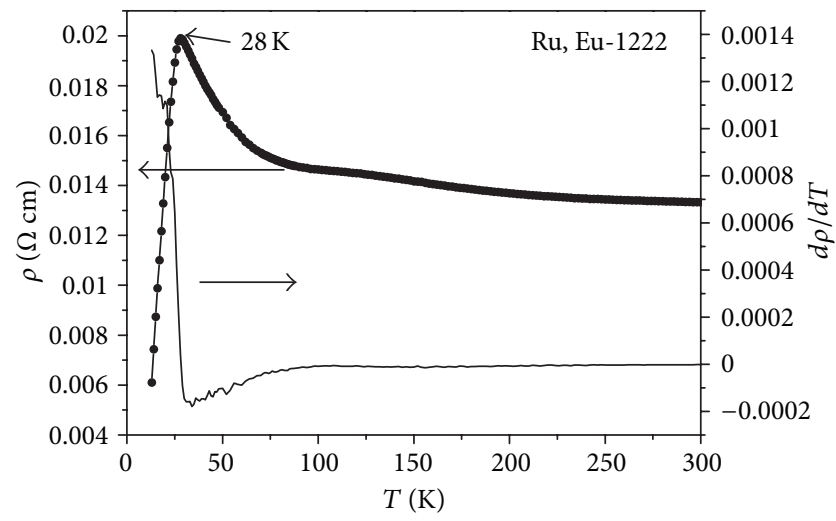

(b)

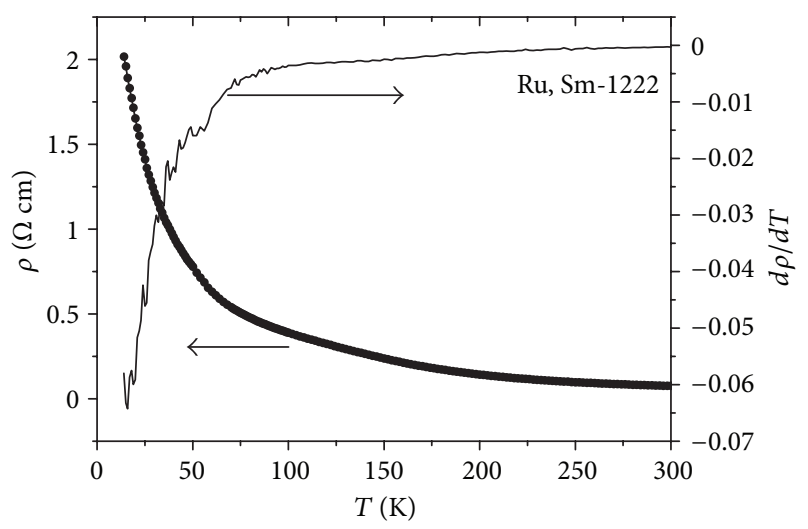

(c)

FIgure 3: $\rho$-T plot along with $d \rho / d T$ of as-prepared samples of (a) $\mathrm{Ru}, \mathrm{Gd}-1222$ (b) Ru, Eu-1222, and (c) Ru, Sm-1222.

The resistivity behavior of $\mathrm{Ru}, \mathrm{Gd}-1222$ and $\mathrm{Ru}, \mathrm{Eu}-1222$ with and without annealing is presented in Figure 6. In the case of $\mathrm{Ru}, \mathrm{Gd}-1222$, the resistivity decreases substantially as a result of annealing in oxygen. This shows that the asprepared samples are oxygen deficient or hole deficient (current carriers in cuprate superconductor). With annealing, the oxygen content/number of holes increases in the samples. The onset superconducting transition temperature of annealed sample increases to $45 \mathrm{~K}$, and zero resistivity

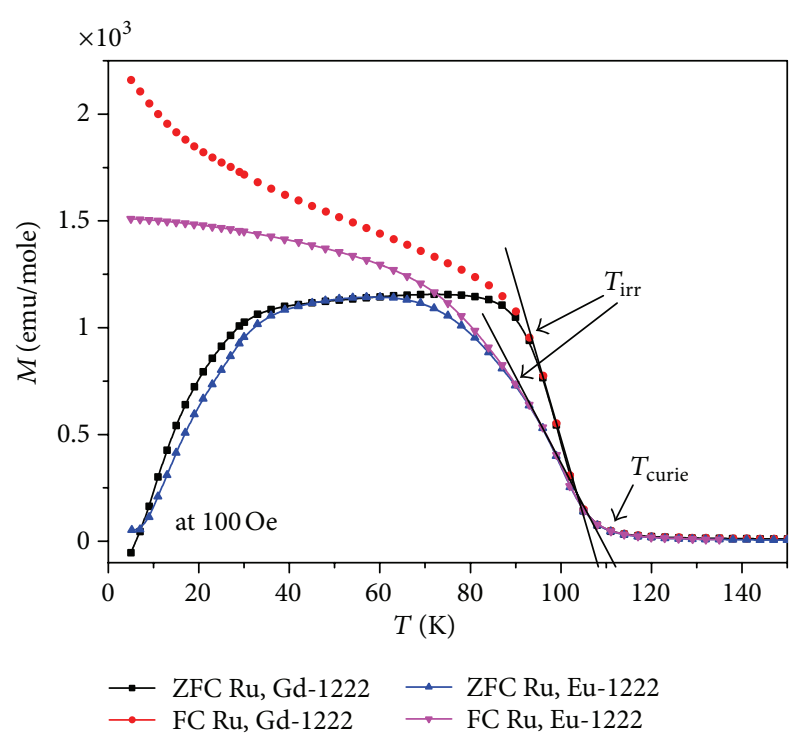

FIgURE 4: Magnetization $(M)$ versus temperature $(T)$ behavior of $\mathrm{Ru}, \mathrm{Gd}-1222$ and Ru, Eu-1222 samples.

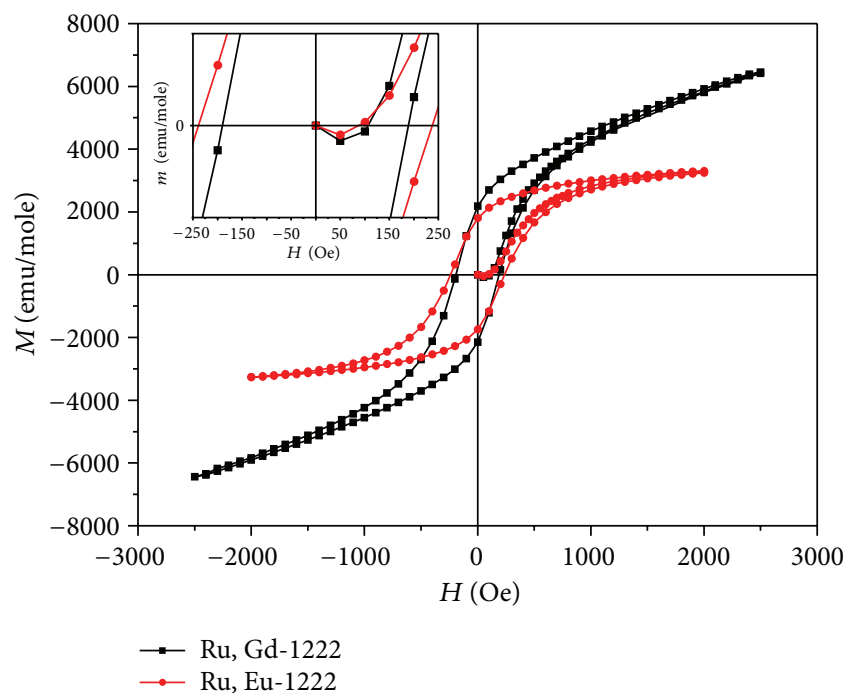

Figure 5: $M-H$ behavior of Ru, Gd-1222 and Ru, Eu-1222 samples at $5 \mathrm{~K}$ showing magnified image at low magnetic field shows clear diamagnetic state.

$T_{c 0}$ becomes $26 \mathrm{~K}$. But in the case of $\mathrm{Ru}, \mathrm{Eu}-1222$, there is a small decrease in normal state resistivity. The onset superconducting transition temperature of annealed sample increases to $30 \mathrm{~K}$. $T_{c 0}$ could not be observed down to $13 \mathrm{~K}$. This shows that though the number of holes was increased due to annealing, this could not overcome the increased separation between the $\mathrm{CuO}_{2}$ planes. From the previous observation, one can say that $\mathrm{Ru}$-1222-type superconductors are oxygen deficient and their oxygen stoichiometry can be varied with the help of annealing, which is a typical nature of cuprate superconductors. 


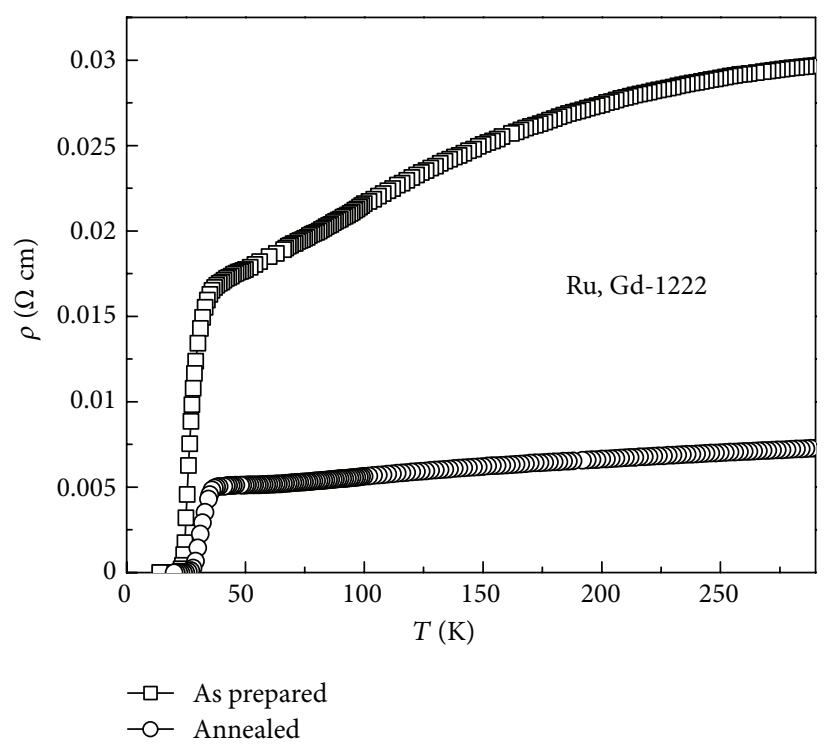

(a)

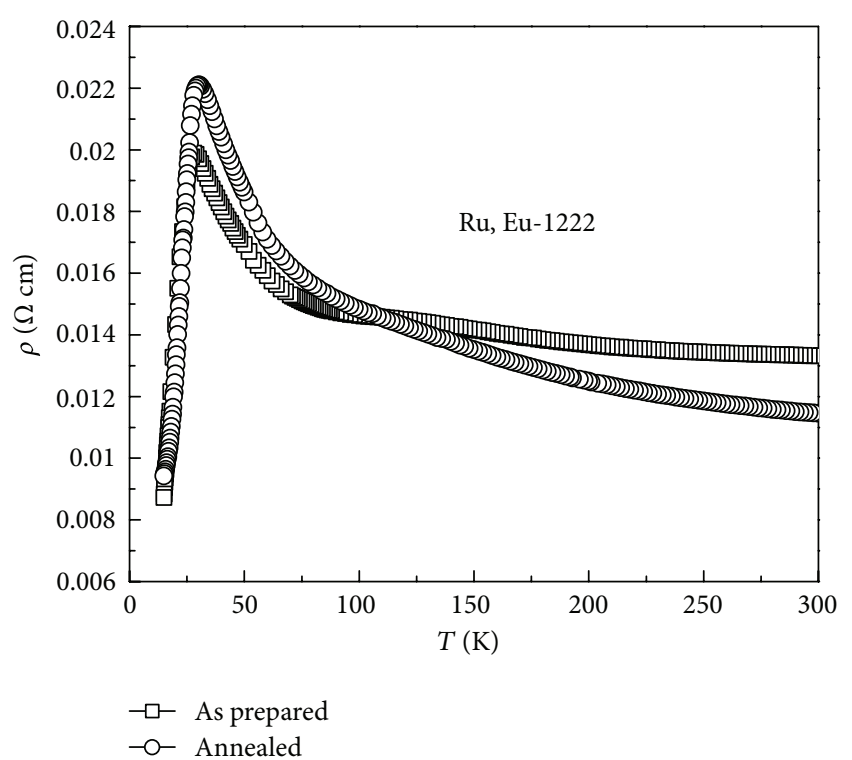

(b)

FIGURE 6: Comparison of the temperature dependence of resistivity of asprepared and annealed samples of (a) Ru, Gd-1222 and (b) Eu, Gd-1222 respectively.

\section{Conclusions}

The $\mathrm{RuSr}_{2} \mathrm{R}_{1.4} \mathrm{Ce}_{0.6} \mathrm{Cu}_{2} \mathrm{O}_{10}$ (where $\mathrm{R}=\mathrm{Gd}$, Eu, and $\mathrm{Sm}$ ) samples have been synthesized through solid-state reaction. XRD analysis revealed that the prepared samples are monophasic in nature. The variation in lattice parameter " $a$ " is proportional to the ionic radii of $\mathrm{Gd}, \mathrm{Eu}$, and $\mathrm{Sm}$. The SEM images clearly show the different grain structure of these compounds. Ru, Gd-1222 has needles type of grains of different sizes imbedded in each other. Ru, Eu-1222 has grains with smooth surface. Ru, Sm-1222 has spongetype porous grain structures. The resistivity-temperature behaviour shows the asprepared sample of Ru, Gd-1222 which shows metallic normal state resistivity before superconducting transition with onset at $36 \mathrm{~K}$ and $T_{c 0}$ (zero resistivity temperature) at $25 \mathrm{~K}$. The as-prepared sample of $\mathrm{Ru}$, Eu1222 show semiconducting normal state resistivity before superconducting transition with onset at $28 \mathrm{~K}$. But the zero resistivity could not be observed in this sample down to $13 \mathrm{~K}$, while $\mathrm{Ru}, \mathrm{Sm}-1222$ is purely semiconducting without any hint of superconductivity. The magnetization- $(M-)$ temperature (T) measurement reveals magnetic transitions $\left(T_{\text {mag }}\right)$ at $105 \mathrm{~K}$. The magnetization $(M)$ versus field $(H)$ hysteresis at $5 \mathrm{~K}$ showed the ferromagnetic behavior of the samples. On comparing the RT behavior of annealed and sintered sample, we can conclude that Ru-1222-type superconductors are oxygen deficient and their oxygen stoichiometry can be varied with the help of annealing, which is a typical nature of cuprate superconductors.

\section{References}

[1] J. Bardeen, L. N. Cooper, and J. R. Schrieffer, "Microscopic theory of superconductivity," Physical Review, vol. 106, no. 1, pp. 162-164, 1957.
[2] I. Felner, U. Asaf, Y. Levi, and O. Millo, "Coexistence of magnetism and superconductivity in $\mathrm{R}_{1.4} \mathrm{Ce}_{0.6} \mathrm{RuSr}_{2} \mathrm{Cu}_{2} \mathrm{O}_{10-\delta}$ (R=Eu and Gd)," Physical Review B, vol. 55, no. 6, pp. R3374R3377, 1997.

[3] L. Bauernfeind, W. Widder, and H. F. Braun, "Rutheniumbased layered cuprates $\mathrm{RuSr}_{2} \mathrm{LnCu}_{2} \mathrm{O}_{8}$ and $\mathrm{RuSr}_{2}\left(\operatorname{Ln}_{1+x} \mathrm{Ce}_{1-x}\right)$ $\mathrm{Cu}_{2} \mathrm{O}_{10}$ (LnSm, Eu and Gd)," Physica C, vol. 254, no. 1-2, pp. 151-158, 1995.

[4] C. Bernhard, J. L. Tallon, Ch. Niedermayer et al., "Coexistence of ferromagnetism and superconductivity in the hybrid ruthenate-cuprate compound $\mathrm{RuSr}_{2} \mathrm{GdCu}_{2} \mathrm{O}_{8}$ studied by muon spin rotation and dc magnetization," Physical Review B, vol. 59, no. 21, pp. 14099-141107, 1999.

[5] O. I. Lebedev, G. Van Tendeloo, G. Cristiani, H.-U. Habermeier, and A. T. Matveev, "Structure-properties relationship in ferromagnetic superconducting $\mathrm{RuSr}_{2} \mathrm{GdCu}_{2} \mathrm{O}_{8}$," Physical Review B, vol. 71, no. 13, Article ID 134523, 2005.

[6] R. Mohan, N. K. Gaur, S. Bhattacharya, and S. K. Gupta, "Crystal growth of $\mathrm{RuSr}_{2} \mathrm{GdCu}_{2} \mathrm{O}_{8}$ compound," Journal of Optoelectronics and Advanced Materials, vol. 4, pp. 1740-1742, 2010.

[7] R. Mohan, K. Singh, N. Kaur et al., "Resistivity study of $\mathrm{RuSr}_{2} \mathrm{GdCu}_{2} \mathrm{O}_{8}$ superconductor," Physica Status Solidi A, vol. 207, no. 2, pp. 411-416, 2010.

[8] E. B. Sonim and I. Felner, "Spontaneous vortex phase in a superconducting weak ferromagnet," Physical Review B, vol. 57, pp. 14000-14003, 1998.

[9] C. A. Cardoso, F. M. Araujo-Moreira, V. P. S. Awana et al., "Spin glass behavior in $\mathrm{RuSr}_{2} \mathrm{Gd}_{1.5} \mathrm{Ce}_{0.5} \mathrm{Cu}_{2} \mathrm{O}_{10-\delta}$," Physical Review $B$, vol. 67, no. 2, Article ID 020407, 4 pages, 2003.

[10] V. P. S. Awana, M. Karppinen, H. Yamauchi, M. Matvejeff, R. S. Liu, and L.-Y. Jang, "Oxygen content and valence of $\mathrm{Ru}$ in $\mathrm{RuSr} 2\left(\mathrm{Gd}_{0.75} \mathrm{Ce}_{0.25}\right)_{2} \mathrm{Cu}_{2} \mathrm{O}_{10-\delta}(\mathrm{Ru}-1222)$ magnetosuperconductor," Journal of Low Temperature Physics, vol. 131, no. 5-6, pp. 1211-1216, 2003. 
[11] Z. Sun, S. Y. Li, Y. M. Xiong, and X. H. Chen, "Preparation, structure and superconductivity of Ru1222 and Ta-doped Ru1212," Physica C, vol. 349, no. 3-4, pp. 289-294, 2001.

[12] W. K. Yeoh, C. A. Kek, and R. A. Shukor, "Superconductivity in ruthenium-based layered cuprate $\mathrm{RuSr}_{2}\left(\mathrm{Gd}_{2-x} \mathrm{Ce}_{x}\right) \mathrm{Cu}_{2} \mathrm{O}_{z}$," Superconductor Science and Technology, vol. 15, no. 3, article 361, 2002.

[13] I. Felner, U. Asaf, Y. Levi, and O. Millo, "Tuning of the superconducting and ferromagnetic behavior by oxygen and hydrogen in $\mathrm{Eu}_{1.5} \mathrm{Ce}_{0.5} \mathrm{RuSr}_{2} \mathrm{Cu}_{2} \mathrm{O}_{10-\delta}$, Physica C, vol. 334, no. 3, pp. 141-151, 2000.

[14] http://www.ccp14.ac.uk/tutorial/lmgp/celref.htm.

[15] R. D. Shannon, "Revised effective ionic radii and systematic studies of interatomic distances in halides and chalcogenides ," Acta Crystallographica A, vol. 32, no. 5, pp. 751-767, 1976.

[16] R. Nigam, A. V. Pan, and S. X. Dou, "Coexistence of ferromagnetism and cluster glass state in superconducting ferromagnet $\mathrm{RuSr}_{2} \mathrm{Eu}_{1.5} \mathrm{Ce}_{0.5} \mathrm{Cu}_{2} \mathrm{O}_{10-\delta}$," J. Appl. Phys, vol. 105, no. 7, Article ID 07E303, 3 pages, 2009. 

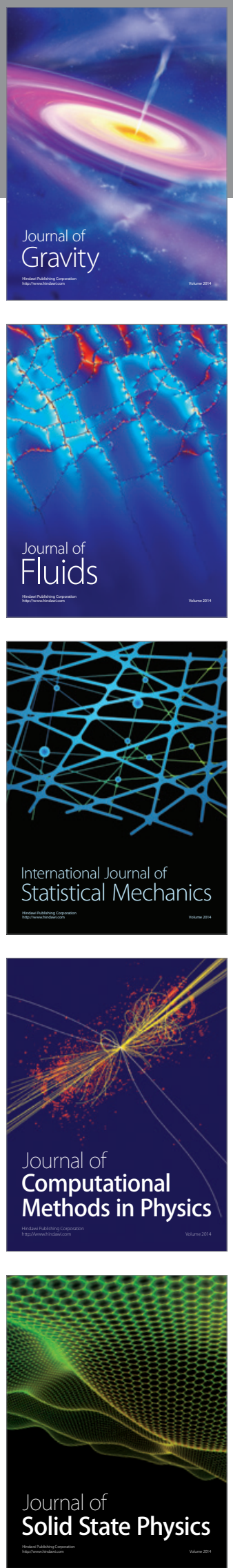

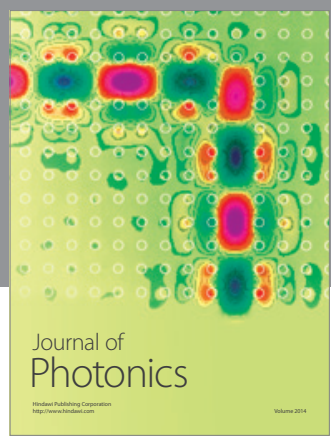

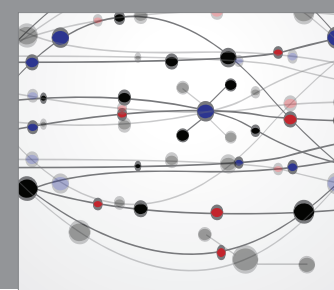

The Scientific World Journal

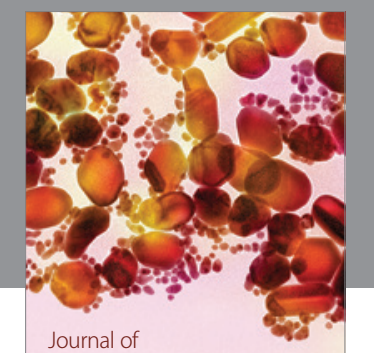

Soft Matter
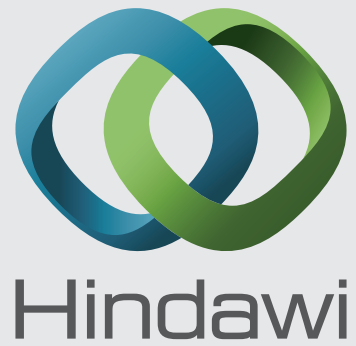

Submit your manuscripts at

http://www.hindawi.com
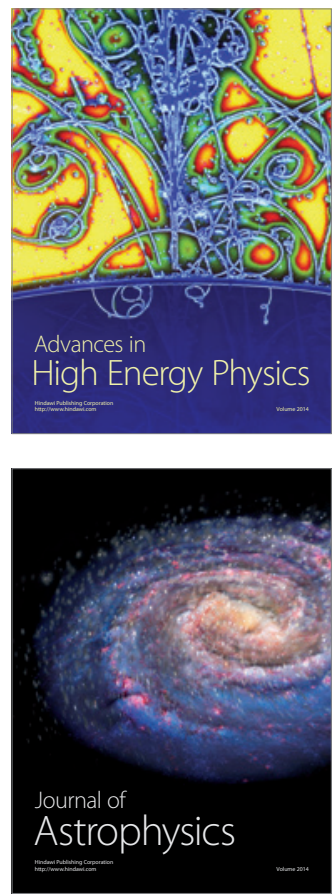
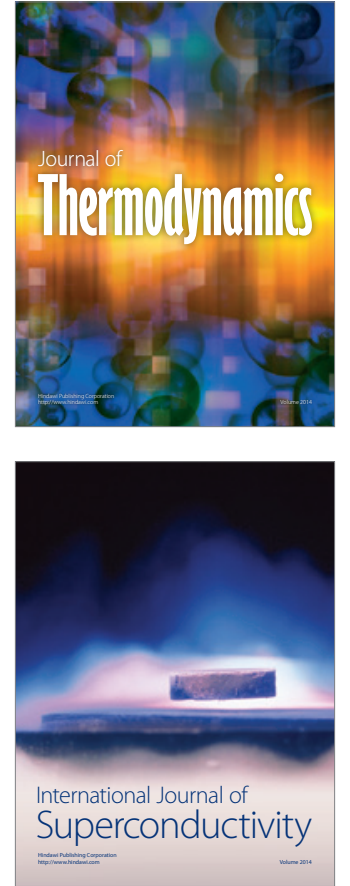
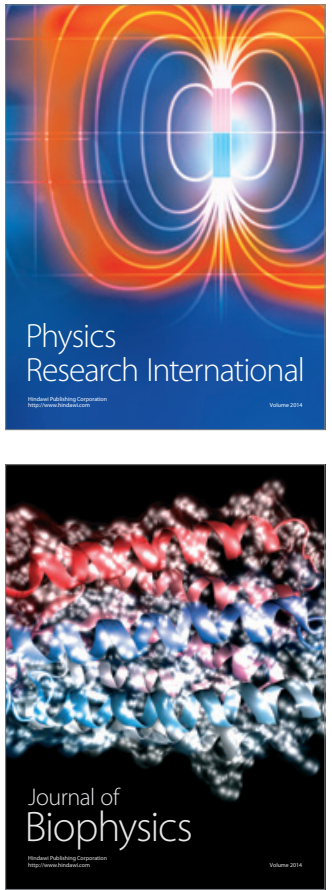
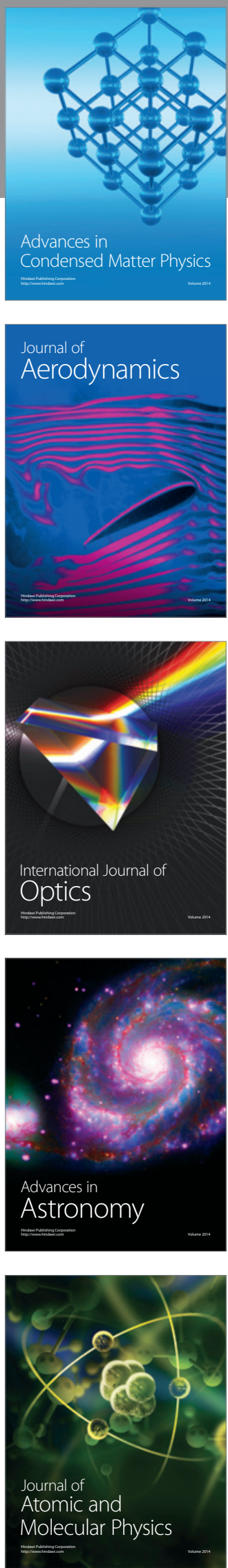